\title{
Continuum Theory of Tkachenko Modes in Rotating Bose-Einstein Condensate
}

\author{
E.B. Sonin \\ Racah Institute of Physics, Hebrew University of Jerusalem, Jerusalem 91904, Israel
}

(Dated: September 29, 2018)

\begin{abstract}
The present paper suggests the continuum theory of Tkachenko modes in a rotating 2D BoseEinstein condensate taking into account density inhomogeneity and compressibility of the condensate. The problem requires solution of coupled hydrodynamic equations for vortex and liquid motion with proper boundary conditions, which were derived for the condensate described by the ThomasFermi approximation. Compressibility becomes essential at rapid rotation with angular velocity close to the trap frequency. The theory is in a reasonable agreement with experimental observation of Tkachenko modes.

PACS numbers: $03.75 . \mathrm{Kk}, 67.40 . \mathrm{Vs}$
\end{abstract}

Tkachenko mode [1] is a transverse sound wave in a vortex lattice, which exists in the ground state of a rotating superfluid. It has attracted a lot of attention in vortex dynamics of laboratory and astrophysical superfluids. Observation of this extremely soft mode (of order or less than a few $\mathrm{Hz}$ ) in laboratory superfluids was very difficult because even slight pinning of vortex ends shades the contribution of vortex shear rigidity transforming the Tkachenko wave into a classical inertial wave [2]. However, discovery of Bose-Einstein condensate (BEC) and possibility to rapidly rotate it made observation of Tkachenko modes more feasible, which resulted in clear experimental detection of them 3]. As well as the Kelvin mode, also possible in the BEC [5], the Tkachenko mode is a direct manifestation of the quantum vorticity since it depends on the circulation quantum.

In a number of aspects external conditions for Tkachenko waves in BEC are essentially different from those in "old" superfluids. First, in a rapidly rotating BEC one cannot consider a superfluid to be incompressible. Effect of finite compressibility was investigated theoretically in the past [2] since the effect is crucial for the long-wavelength limit of the Tkachenko mode. But at that time it was considered as a theoretical curiosity since in order to reveal it one needed containers about a few hundred meters diameter. In the BEC case the situation is essentially different because in contrast to a strongly interacting bose liquid as He II, BEC is a weakly interacting bose gas with very low sound speed and very high compressibility. Importance of high liquid compressibility for Tkachenko waves in BEC was pointed out by Baym [4], who rederived the spectrum of Tkachenko waves in a compressible liquid [6] known from Ref. 2 and compared it with the experiment [3]. Another important feature of rotating BEC, also connected with its high compressibility, is that the liquid density is essentially inhomogeneous. This feature was taken into account in the theory by Anglin and Crescimanno 7] within the frame of the continuum theory, which replaces a discrete vortex lattice by a continuous medium, like the elasticity theory for atomic crystals. But they neglected liquid com- pressibility, while a proper comparison with the experiment requires a theory taking into account the both features, compressibility and inhomogeneity. Recently the Tkachenko mode in rotating BEC was investigated numerically with solving the equations of Gross-Pitaevskii theory (mean-field theory) [8, 9]. The numerical results well agreed with the experiment. But numerical calculations, whatever powerful and useful they can be, do not exclude necessity to develop the analytical approach, as far as it possible, since the latter gives analytical and scaling properties and therefore provides a deeper insight into physics of the phenomenon. In the experiment there are good conditions for application of a continuum theory since usually the relevant length scale (the condensate size) essentially exceeded the intervortex distance. The goal of the present paper is to suggest the continuum theory of Tkachenko modes in a rapidly rotating BEC taking into account liquid compressibility and inhomogeneity. The main challenge for the theory was to formulate proper boundary conditions for oscillating BEC.

Let us remind the equations of motion of a homogeneous compressible superfluid at $T=0$ (no normal component) in the rotating coordinate frame [2]:

$$
\begin{gathered}
\frac{\partial \rho^{\prime}}{\partial t}+\rho_{0} \vec{\nabla} \cdot \vec{v}=0 \\
\rho_{0}\left(\frac{\partial \vec{v}}{\partial t}+2 \vec{\Omega} \times \vec{v}_{L}\right)=-c_{s}^{2} \vec{\nabla} \rho^{\prime}, \\
\rho_{0} \vec{\kappa} \times\left(\vec{v}_{L}-\vec{v}\right)=\frac{\kappa c_{T}^{2}}{2 \Omega}[2 \vec{\nabla}(\vec{\nabla} \cdot \vec{u})-\Delta \vec{u}] .
\end{gathered}
$$

Here $\rho^{\prime}(\vec{r})$ is the oscillating component of the liquid mass density $\rho(\vec{r})=\rho_{0}+\rho^{\prime}(\vec{r})$ around the equilibrium homogeneous density $\rho_{0}, \vec{u}$ is the vortex displacement, $\vec{v}_{L}=d \vec{u} / d t$ is the vortex velocity, $\vec{v}$ is the liquid velocity averaged over the vortex-lattice cell, $\vec{\Omega}$ is the angular velocity, $c_{s}$ is the sound velocity, $\kappa=h / m$ is the circulation quantum, and $c_{T}=\sqrt{\kappa \Omega / 8 \pi}$ is the Tkachenko-wave velocity. Assuming that $c_{T} \ll c_{s}$ and $c_{T} k \ll \Omega$ one receives 
for the spectrum of plane waves $\propto \exp (i \vec{k} \cdot \vec{r}-i \omega t)$ the dispersion relation:

$$
\omega^{4}-\omega^{2}\left(4 \Omega^{2}+c_{s}^{2} k^{2}\right)+c_{s}^{2} c_{T}^{2} k^{4}=0,
$$

which yields the gapped sound mode

$$
\omega^{2}=4 \Omega^{2}+c_{s}^{2} k^{2}
$$

and the soft quantum mode (see Ref. 10 and Eq. (4.73) in Ref. 2)

$$
\omega^{2}=\frac{c_{T}^{2} c_{s}^{2} k^{4}}{4 \Omega^{2}+c_{s}^{2} k^{2}} .
$$

So for the soft (Tkachenko) mode compressibility is essential in the long-wavelength limit $k \ll \Omega / c_{s}$. It transforms the Tkachenko wave with the sound spectrum $\omega=c_{T} k$ to a softer mode $\omega \propto k^{2}$. In the Tkachenko mode motion of both the vortex lattice and the liquid has an elliptic polarization but longitudinal components parallel to the wave vector $\vec{k}$ are very small. The transverse components (normal to $\vec{k}$ ) of the vortex lattice and the liquid are close one to another: $v_{L t} \approx v_{t}$.

Generalization of these equations onto an inhomogeneous liquid with equilibrium density $\rho_{0}(\vec{r})$, which varies in the plane normal to the rotation axis. is straightforward. The goal of this paper is to consider only axisymmetric Tkachenko eigenmodes in a axisymmetric rotating BEC. Thus we write the equations of motion in the polar system of coordinates for the monochromatic mode $\propto e^{-i \omega t}$ :

$$
\begin{gathered}
2 \Omega i \omega v_{r}=-\omega^{2} v_{t}-\frac{c_{T}^{2}}{\rho_{0}} \frac{1}{r^{2}} \frac{\partial}{\partial r}\left[\rho_{0} r^{3} \frac{\partial}{\partial r}\left(\frac{v_{t}}{r}\right)\right], \\
2 \Omega i \omega v_{t}=\frac{c_{s}^{2}}{\rho_{0}} \frac{\partial}{\partial r}\left[\frac{1}{r} \frac{\partial\left(\rho_{0} r v_{r}\right)}{\partial r}\right] .
\end{gathered}
$$

We excluded all variables except for the tangential (azimuthal in polar coordinates) component of the velocity $v_{t} \approx v_{L t}$ and the radial component of the liquid velocity $v_{r}$. The latter, though being much smaller than $v_{t}$, is crucial for the compressibility effect. For a weakly interacting bose gas $c_{s}^{2}$ is proportional to the density $\rho_{0}$. Therefore the ratio $c_{s}^{2} / \rho_{0}$ can be replaced by its value $c_{s}^{2}(0) / \rho_{0}(0)$ in the BEC center $r=0$.

Let us remind the results for Tkachenko eigenmodes in an incompressible $\left(c_{s} \rightarrow \infty\right)$ homogeneous ( $\rho_{0}=$ const) liquid in a cylindric container. Then the radial liquid velocity $v_{r}$ vanishes, and Eq. (77) is the Bessel equation with a solution $v_{t} \propto J_{1}(k r)$. The eigenvalues of the wave number $k$ are determined from the boundary condition at $r=R$, where $R$ is the radius of the vortex-lattice sample, which normally is close to the container radius. In the ideal axisymmetric case without any interaction of vortices with lateral walls the radial flux of the transverse momentum of the vortex lattice, which is given by the corresponding component of the lattice stress tensor, must vanish. This gives the boundary condition

$$
\frac{d u(R)}{d r}-\frac{u(R)}{R}=-\frac{1}{i \omega}\left[\frac{d v_{t}(R)}{d r}-\frac{v_{t}(R)}{R}\right]=0 .
$$

The flux of the angular moment into the liquid is proportional to the same stress-tensor component and therefore vanishes also. Equation (9) leads to the condition $J_{2}(k R)=0$ imposed on the wave numbers $k$. Traditionally in the papers on BEC they scale the Tkachenko-mode frequencies by the frequency $\Omega b / R$, where $b=\sqrt{\kappa / \sqrt{3} \Omega}$ is the intervortex distance [3, 7, 9]:

$$
\omega=\tilde{\omega} \frac{\Omega b}{R}=\tilde{\omega} \sqrt{\frac{8 \pi}{\sqrt{3}}} \frac{c_{T}}{R} .
$$

Then the first two roots $k R=5.14$ and 8.42 of the equation $J_{2}(k R)=0$ give the first two reduced eigenfrequencies of the Tkachenko mode $\tilde{\omega}=\sqrt{\sqrt{3} / 8 \pi} k R=0.263 k R$ with the ratio $8.42 / 5.14=1.64$. Another interesting case is the limit of strong interaction of vortices with rough lateral walls, when $v_{t}(R)=0$. Then the eigenfrequencies are given by two first roots of the equation $J_{1}(k R)=0$ $k R=3.83$ and 7.02 with the ratio of the two lowest eigenfrequencies $7.02 / 3.83=1.83$.

Now let us consider an inhomogeneous (but still incompressible) liquid. The analysis will be applied to the rotating $2 \mathrm{D}$ BEC in pancake geometry with the density profile $\rho_{0}=\rho(0)\left(1-r^{2} / R^{2}\right)$ determined from the ThomasFermi approximation [11]. Here $R=\sqrt{2} c_{s}(0) / \sqrt{\omega_{\perp}^{2}-\Omega^{2}}$ is the BEC radius (Thomas-Fermi radius), $c_{s}(0)$ is the sound velocity at the symmetry axis $r=0$, and $\omega_{\perp}$ is the trap frequency, which characterizes the curvature of the trapping parabolic potential [3]. Since the momentum flux is proportional to $\rho_{0}$ and the latter vanishes at $r=R$, it looks that the flux through the boundary vanishes independently on whether the boundary condition Eq. (9) is satisfied or not and the latter is unnecessary. But this is not the case as one can see from solution of the equation of motion close to $r=R$ by expansion in small $(R-r) / R$. With the relative error of the order of $(R-r)^{2} / R^{2}$ the general solution of Eq. (7) is $v_{t} \approx r\left[C_{1}+C_{2} \ln (R-r)\right]$, where $C_{1}$ and $C_{2}$ are arbitrary constants. The component $\propto C_{2}$ gives a divergent contribution to the displacements and a finite contribution to the stress tensor despite the factor $\rho_{0} \propto R-r$. This would violate the conservation law for the angular moment and this component should be absent. This requirement is satisfied if the boundary condition Eq. (9) takes place. Therefore the condition Eq. (9) is relevant also for an inhomogeneous liquid with $\rho_{0}(r) \rightarrow 0$ at the border. The equation of motion for an inhomogeneous liquid has no analytic solution in the whole interval of $r$ but it is not difficult using Mathematica to find the eigenmodes. First two of them are $\tilde{\omega}=1.428$ and 2.327 with the ratio of 
the two lowest eigenfrequencies $2.327 / 1.428=1.63$. This agrees with the results of Ref. 7 .

According to Eq. (6) the compressibility effect becomes important if $k$ is of the order or less than $\Omega / c_{s}$. Since the eigenvalues of $k$ are of the order of $1 / R$ this yields the condition for a strong compressibility effect: $\Omega R / c_{s}(0) \sim \Omega / \sqrt{\omega_{\perp}^{2}-\Omega^{2}} \gg 1$. Thus at rapid rotation of the BEC with angular velocity $\Omega$ close to the trap velocity $\omega_{\perp}$. liquid compressibility should be taken into account. If we want to make the quantitative analysis for an incompressible liquid, we should solve the system of two coupled second-order differential equations (77) and (8) with proper boundary conditions. One of them remains to be Eq. (9), but we need also the boundary condition imposed on the radial liquid velocity $v_{r}$. We use the arguments similar to those used for derivation of Eq. (9). The total mass balance requires that the radial mass current $\rho_{0}(r) v_{r}(r)$ at the BEC border $r=R$ vanishes. Indeed, even though the BEC border is mobile because of the oscillation, the total mass, which can be transferred through the equilibrium border $r=R$, is a second-order quantity with respect to an oscillation amplitude. The radial mass current vanishes for any finite radial velocity $v_{r}(R)$ since $\rho(r) \rightarrow 0$ at $r \rightarrow R$. But some condition is required to provide $v_{r}(R)$ to be finite. In order to derive this condition we solve Eq. (8) at $r \approx R$ by series expansion [neglecting terms $\sim(R-r)^{2}$ ]:

$v_{r}(r)=\frac{\Omega i \omega v_{t}(R) R}{c_{s}(0)^{2}} \frac{R-r}{2}+C_{1}\left(1+\frac{R-r}{R}\right)+\frac{C_{2}}{R-r}$.

The divergent component $\propto C_{2}$ gives a finite mass flow at the border and should be ruled out. Taking a derivative from this expression and excluding the constant $C_{1}$ we receive the boundary condition imposed on $v_{r}$ :

$$
\frac{d v_{r}(R)}{d r}+\frac{v_{r}(R)}{R}=-\frac{i \omega \Omega R}{2 c_{s}(0)^{2}} v_{t}(R) .
$$

The left-hand side of the boundary condition (12) is the divergence $\vec{\nabla} \cdot \vec{v}$ of the liquid velocity in polar coordinates. The divergence remains constant at the boundary of the BEC sample, but the density $\rho_{0}(R)$ vanishes there. As a result, the mass continuity equation yields that

$$
\frac{\partial \rho}{\partial t}=-\vec{\nabla} \cdot\left(\rho_{0} \vec{v}\right)=-\vec{v} \cdot \vec{\nabla} \rho_{0} .
$$

Thus at the sample boundary the whole variation of the density originates from the oscillation of the boundary with the velocity $v_{r}(R)$. The same boundary condition is valid for other compressional modes of the BEC [12, 13].

For better understanding of parametric dependences of the eigenfrequencies it is useful to introduce the dimensionless radius $z=r / R$ and to scale the radial velocity, introducing the velocity $u: v_{r}=i u c_{T} / c_{s}(0)$. Then the system of equations is:

$$
s q\left(1-z^{2}\right) u(z)=q^{2}\left(1-z^{2}\right) v_{t}(z)
$$

$$
\begin{aligned}
& +\frac{1}{z^{2}} \frac{d}{d z}\left\{\left(1-z^{2}\right) z^{3} \frac{d}{d z}\left[\frac{v_{t}(z)}{z}\right]\right\}, \\
& s q v_{t}(z)=\frac{d}{d z}\left\{\frac{1}{z} \frac{d\left[\left(1-z^{2}\right) z u(z)\right]}{d z}\right\},
\end{aligned}
$$

where $q$ determines the reduced frequency, $\tilde{\omega}=0.263 q$, and the parameter

$$
s=\frac{2 \Omega R}{c_{s}(0)}=\frac{2 \sqrt{2} \Omega}{\sqrt{\omega_{\perp}^{2}-\Omega^{2}}}
$$

characterizes the effect of compressibility. In the dimensionless form the boundary condition (12) is $d u(1) / d z+$ $u(1)=-s q v_{t}(1) / 4$.

Let us discuss now restrictions on validness of the presented analysis. In order to observe the strong compressibility effect $s \gg 1$ one should rotate the BEC rapidly, at $\Omega \sim \omega_{\perp}$. But if $\Omega$ approaches to $\omega_{\perp}$ close enough at fixed number of atoms the condition of small ratio $c_{T} / c_{s}$ is violated. Since the vortex core size is $\xi \sim \kappa / c_{s}$, the large ratio $c_{T} / c_{s} \sim \xi / b$ means that the vortex cores start to overlap. This signals that we approach to the critical angular velocity, similar to the upper critical magnetic field $H_{c 2}$, when rotation should suppress the superfluid order parameter. When $\xi \sim b$ the properties of the BEC become essentially different from those assumed in the present work (see Ref. 14 and references therein). However with a number of atoms large enough one can reach very high values of $s$ without violation of the condition $\xi / b \ll 1[15$. Another assumption of our analysis was solid body rotation of the BEC with the constant vortex density given by the Feynman formula $n_{v}=2 \Omega / \kappa$. In a inhomogeneous liquid this formula is not exact. Corrections to this formula due to forces from density gradients were found in Refs. 7 and 16 . But these corrections are essential only at the distance of the order $b$ from the sample boundary [14] and therefore are not essential for determination of eigenfrequencies until $R \gg b$.

In order to find the eigenfrequencies we determined numerically (at fixed $s$ ) eigenvalues of $q$ at which Eqs. (14) and (15) have a solution with proper boundary conditions. In addition to the boundary conditions at the BEC boundary discussed above, there are standard conditions in the BEC center that the velocities are not divergent, namely, $v_{r} \propto v_{t} \propto r$. If compressibility is important any reduced eigenfrequency is not a number but a function of $s: \tilde{\omega}_{i}=f_{i}(s)$. At $s \rightarrow 0$ the functions $f_{i}(s)$ are constant, but at large $s$ the functions $f_{i}(s)$ are inversely proportional to $s$. The numerical solution yields that at large $s$ the two first eigenfrequencies are $\tilde{\omega}_{1}=7.17 / \mathrm{s}$ and $\tilde{\omega}_{2}=16.9 / \mathrm{s}$. Figure 1 shows the numerically found first eigenfrequency $\tilde{\omega}_{1}$ plotted as a function of $\Omega / \sqrt{\omega_{\perp}^{2}-\Omega^{2}}=s / 2 \sqrt{2}$ (solid line). The black squares show experimental points [3] plotted in dimensionless variables by I. Coddington. They were obtained 


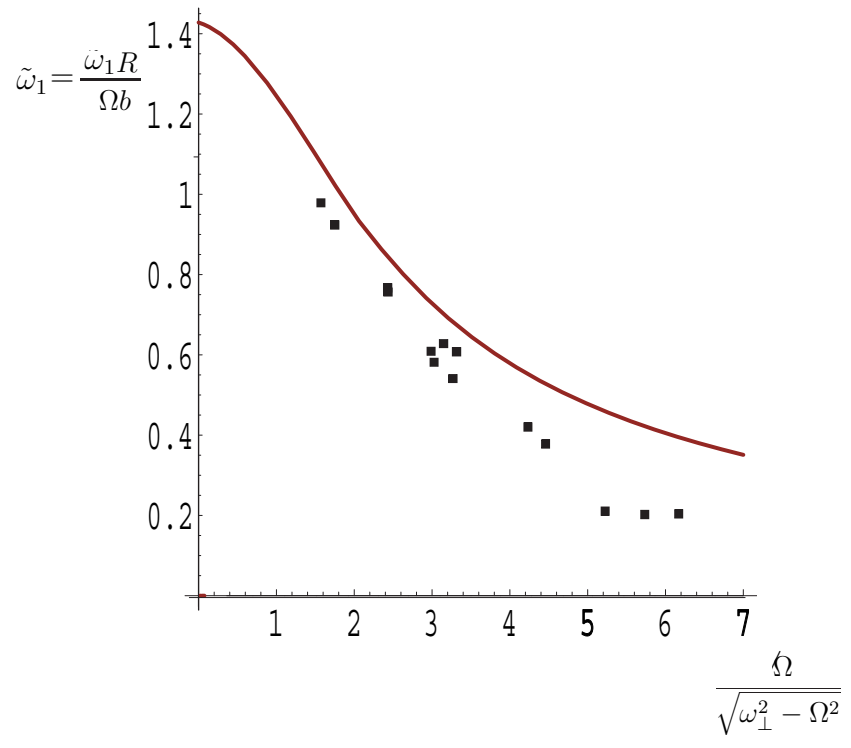

FIG. 1: Comparison between the theory (solid line) and the experiment (black squares).

for various parameters, but collapse on the same curve, as expected from the present analysis. Quantitative agreement between the theory and the experiment looks quite good. It becomes worse at larger $\Omega / \sqrt{\omega_{\perp}^{2}-\Omega^{2}}$, since the growth of the parameter $\xi / b$ in this area makes the theory less accurate (see above). Coddington al. [3] measured also the ratio of the two first frequencies $\omega_{2} / \omega_{1}=1.8$ at $\Omega / \omega_{\perp}=0.95$, which corresponds to $s=8.61$, The present theory predicts the ratio $\omega_{2} / \omega_{1}=2.09$.

In summary the continuum theory of Tkachenko modes was developed, which takes into account the liquid inhomogeneity and finite compressibility. Application of the theory to a rapidly rotating Bose-Einstein condensate required formulation of proper boundary conditions at the condensate border where atom density vanishes. The theory is in good agreement with observations of Tkachenko modes in the rapidly rotating Bose-Einstein condensate.

I thank Ian Coddington who prepared the experimental part of Fig. 11 I also appreciate very much an interesting comment by Marco Cozzini, Lev Pitaevskii, and Sandro Stringari concerning comparison of the present analysis with that based on the compressibility sum rule [6].

[1] V.K. Tkachenko, Zh. Eksp. Teor. Fiz. 94(2), 315-328 (1988) [Sov. Phys.-JETP 23, 1049 (1966)].

[2] E.B. Sonin, Rev. Mod. Phys. 59, 87 (1987).

[3] I. Coddington, P. Engels, V. Schweikhard, and E.A. Cornell, Phys. Rev. Lett. 91, 100402 (2003).

[4] G. Baym, Phys. Rev. Lett. 91, 110402 (2003).

[5] A.L. Fetter, Phys. Rev. A 69, 043617 (2004).

[6] Effect of compressibility on Tkachenko waves in BEC was also discussed using the compressibility sum rule by $\mathrm{M}$. Cozzini, L. P. Pitaevskii, and S. Stringari, 92, 220401 (2004).

[7] J.R. Angin and M. Crescimanno, cond-mat/0210063

[8] T. Mizushima, Y. Kawaguchi, K. Machida, T. Ohmi, T. Isoshima, and M.M. Salomaa, Phys. Rev. Lett. 92, 060407 (2004).

[9] L.O. Baksmaty, S.J. Woo, S. Choi, and N.P. Bigelow, Phys. Rev. Lett. 92, 160405 (2004).

[10] Equations (5) and (6) can be also derived from a more general expression received by G.E. Volovik and V.S. Dotsenko (jr), Pisma Zh. Eksp. Teor. Fiz. 29, 630 (1979) [JETP Lett. 29, 576 (1979)], within the formalism of Poisson brackets.

[11] F. Dalfovo, S. Giorgini, L.P. Pitaevskii, and S. Stringari, Rev. Mod. Phys. 71, 463 (1999).

[12] M. Cozzini and S. Stringari, Phys. Rev. A 67, 041602 (2003).

[13] S. Stock, V. Bretin, F. Chevy, and J. Dalibard, Europhys. Lett. 65, 594 (2004).

[14] I. Coddington, P.C. Haljan, P. Engels, V. Schweikhard, S. Tung, and E.A. Cornell, con-mat/0405240

[15] Baksmaty et al. [9] believe that the parameter, which gauges the effect of compressibility, is $\xi / b$ but not $s=$ $\Omega R / c_{s}$, in contradiction to our analysis.

[16] D.E. Sheehy and L. Radzihovsky, cond-mat/0402637 